\title{
Subcorneal hematoma as an imitator of acral melanoma: Dermoscopic diagnosis
}

\author{
Omer Faruk Elmas, ${ }^{1}$ (i) Necmettin Akdeniz ${ }^{2}$ \\ ${ }^{1}$ Department of Dermatology, Ahi Evran University Faculty of Medicine, Kirsehir, Turkey \\ ${ }^{2}$ Department of Dermatology, Medeniyet University Faculty of Medicine, Istanbul, Turkey
}

\begin{abstract}
OBJECTIVE: There are few studies investigating dermoscopic features of subcorneal hematoma, which is one of the imitators of acral melanoma. In this study, we aimed to describe dermoscopic findings of SH which will facilitate the diagnosis by reducing the use of invasive procedures.

METHODS: This study included patients with subcorneal hematoma. Clinical, demographic and dermoscopic findings of all the patients were retrospectively reviewed. The diagnosis of subungual hematoma was confirmed using a positive scratch test in all of the patients.

RESULTS: The findings showed that red-black $(n=9,45 \%)$ was the most common dermoscopic color followed by brown $(n=4,20 \%)$, red $(n=3,15 \%)$, black $(n=2,10 \%)$, brown-black $(n=1,5 \%)$ and red-brown $(n=1,5 \%)$. The most common pigmentation pattern was homogenous pattern $(n=13,65 \%)$. Eleven (55\%) lesions showed globular pattern and eight (40\%) lesions exhibit parallel ridge pattern. Eight ( $40 \%)$ lesions showed a combination of homogenous and globular patterns. The globules were disconnected from the homogenous pigmentation in six lesions. In two $(10 \%)$ lesions, the globules were localized over the homogenous pigmentation.

CONCLUSION: The presence of homogenous red to brown to black pigmentation combined with globules may lead to the correct diagnosis in a subcorneal hematoma. Scratch test may be a practical and minimal invasive diagnostic option to confirm the diagnosis in suspicious cases. To our knowledge, this is the most comprehensive study focusing on the dermoscopic aspect of the subcorneal hematoma.
\end{abstract}

Keywords: Dermoscopy; subcorneal hematoma; talon nair.

Cite this article as: Elmas OF, Akdeniz N. Subcorneal hematoma as an imitator of acral melanoma: Dermoscopic diagnosis. North Clin Istanb 2020;7(1):56-59.

S ubcorneal hematoma ( $\mathrm{SH})$ means blood accumulation beneath the horny layer of the skin. SH usually occurs in the palmoplantar region as a consequence of blunt trauma. Differential diagnosis of the entity mainly includes acral lentiginous melanoma and acral nevi $[1,2]$.

Dermoscopy is a non-invasive and practical tool that recently has become one of the major diagnostic methods in daily dermatology practice. Dermoscopic features of many dermatological conditions have been well described. However, only one original study focusing on the dermoscopic findings of $\mathrm{SH}$ exists in the respective literature [1].

In this study, we aimed to describe dermoscopic findings of $\mathrm{SH}$, which will facilitate the diagnosis by reducing the use of invasive procedures.

\section{MATERIALS AND METHODS}

This study included patients with subcorneal hematoma. All the patients were selected from the outpatient de- 
partment of a tertiary center between December 2017 and November 2018. Clinical, demographic and dermoscopic findings of all the cases were retrospectively reviewed. Dermoscopic images of each lesion were obtained using a polarized handheld dermoscope (DermLite DL II Pro HR; 3Gen Inc.; San Juan Capistrano, CA). Dermoscopic photographing was performed with a dermoscope adopted camera phone with a high resolution (iPhone 7 plus, Apple, California, USA). The diagnosis of subungual hematoma was confirmed by a positive scratch test in all the cases. The horny layer of the lesional skin has been scraped with a sterile scalpel of the appropriate size, in a parallel direction to the lesion. The test was considered to be positive in case of the complete removal of the discoloration. Descriptive statistical analysis was performed using the SPSS pocket program (SPSS Inc., Chicago, IL). All the procedures followed were in accordance with the Helsinki Declaration and this study was approved by the local clinical research ethics committee (Decision date/number: 13.11.2018/ 2018-21/177).

\section{RESULTS}

This study included 14 men (70\%) and six women (30\%) patients. The mean age of the patients was 41.4 (age range 11-62). The mean duration of the lesions was one month (range 7 days -3 months). The most common localization was soles $(n=12,60 \%)$ followed by palms $(n=5,25 \%)$ and volar surface of the hand $(n=3,15 \%)$. Four patients had a history of trauma, and two patients were on anticoagulant therapy. After dermoscopic examination, a scratch test was performed in all the cases allowing a complete clearance of the pigmentation. Thus, no histopathological examination was needed in any patient.

On the dermoscopic examination, the distribution of the colors was as follows: red-black 9 (45\%) (Fig. 1A, B), brown 4 (20\%) (Fig. 1C), red 3 (15\%) (Fig. 1D), black $2(10 \%)$ (Fig. 1E), brown black $1(5 \%)$ and red-brown 1 (5\%), respectively.

The most common pigmentation pattern was homogenous pattern $(n=13,65 \%)$ (Fig. 1A-E). Eleven (55\%) lesions showed a globular pattern (Fig. 1A, B, E) and eight (40\%) lesions exhibited a parallel ridge pattern (Fig. 1C). Eight (40\%) lesions showed a combination of the homogenous and globular patterns. The globules were disconnected from the homogenous pigmentation in six $(30 \%)$ of the lesions. The globules were localized
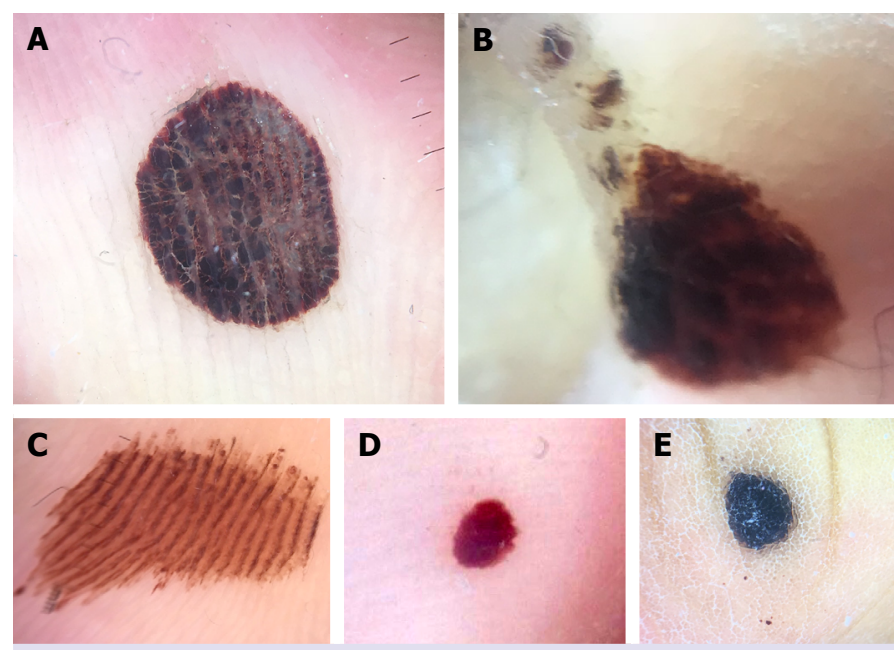

D

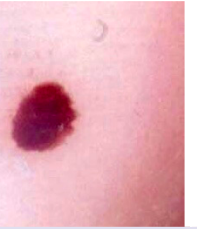

E

FIGURE 1. (A) Homogenous red black pigmentation and globules localized on the homogenous pigmentation, (B) Homogenous red black pigmentation with both satellite globules and globules localized on the homogenous pigmentation, (C) Brown pigmentation on the ridges, (D) Homogenous red pigmentation without globules, (E) Homogenous black pigmentation with satellite globules.

on the homogenous pigmentation in two $(10 \%)$ of the lesions (Fig. 1A, B). The clinic and dermoscopic features of the cases were detailed in Table 1 .

\section{DISCUSSION}

SH describes a traumatic rupture of the dermal papillary capillaries resulting extravasation of red blood cells into stratum corneum of the skin [1]. Many other terms, including intracorneal hematoma [2], talon nair [3], black heel [4], basketball heel [5], hyperkeratosis hemorrhagica [6] and tennisferse [7], have been used to define this condition. It is not always easy to diagnose $\mathrm{SH}$ correctly by unaided eye. Acral melanoma is the main differential diagnosis of the entity $[1,2]$. Acral nevi, pyogenic granuloma, angiokeratoma and even plantar warts should also be in the differential diagnosis list [1]. In the present study, 5 and 4 lesions had a preliminary diagnosis of melanoma and acral melanocytic nevus, respectively.

$\mathrm{SH}$ may occur as a result of both sudden trauma and repetitive microtraumas. Robertson et al. reported a case of $\mathrm{SH}$ associated with repetitive traumas from a Sony PlayStation $^{\circledR} 3$ (Sony Corporation, Tokyo, Japan) vibration feedback controller [8].

Dermoscopic features of $\mathrm{SH}$ were first described in Saida et al's study. The authors suggested the term "peb- 
TABLE 1. Clinical and dermoscopic findings of the cases with subcorneal hematoma

\begin{tabular}{lcccccc} 
Case $(\mathrm{n}=20)$ & Clinical diagnosis & $\begin{array}{c}\text { Scratch Test } \\
(\mathrm{n}=20,100 \%)\end{array}$ & Color & $\begin{array}{c}\text { Homogenous pattern } \\
(\mathrm{n}=13,65 \%)\end{array}$ & $\begin{array}{c}\text { Globular pattern } \\
(\mathrm{n}=11,55 \%)\end{array}$ & $\begin{array}{c}\text { Parallel ridge pattern } \\
(\mathrm{n}=8,40 \%)\end{array}$ \\
\hline 1 & Hematoma & + & Black & + & + & - \\
2 & Hematoma & + & Red-black & + & + & + \\
3 & Nevus & + & Red-black & & + & - \\
4 & Hematoma & + & Brown & - & - & + \\
5 & Hematoma & + & Red & + & + & + \\
6 & Melanoma & + & Red-brown & + & + & - \\
7 & Hematoma & + & Brown & - & + & - \\
8 & Melanoma & + & Red-black & + & - & + \\
9 & Hematoma & + & Red-black & + & + & - \\
10 & Nevus & + & Red-black & + & + & + \\
11 & Melanoma & + & Brown & - & + & + \\
12 & Hematoma & + & Red-black & + & + & + \\
13 & Nevus & + & Brown black & - & + & + \\
14 & Hematoma & + & Red-black & + & + & + \\
15 & Hematoma & + & Brown & - & + & + \\
16 & Hematoma & + & Red & + & + & - \\
17 & Melanoma & + & Red-black & - & + & - \\
18 & Nevus & + & Red & + & + & + \\
19 & Melanoma & + & Red-black & + & + & +
\end{tabular}

bles on the ridges" to define reddish-black droplets distributed on the ridges [9].

The most comprehensive study focusing on the subject is the study of Zalaudek et al. in which the authors described dermoscopic features of the 15 lesions of $\mathrm{SH}$. They revealed that the most common color of hematomas was red-black, followed by brown to black [1]. The red-black color was also demonstrated to be the most common color in the present study. In Zalaudek et al's study, the most common pigmentation pattern was homogenous pattern (53.3\%) followed by globular $(46.7 \%)$ and parallel ridge patterns (40\%) [1]. In our study, the most common pigmentation pattern was also a homogenous pattern (65\%) followed by globular (55\%) and parallel ridge (40\%) patterns, similarly. Zalaudek et al. also identified fibrillar and furrow patterns in two patients. In our study, none of the patients showed these rare patterns [1]. The frequencies of the dermoscopic findings observed in the present study and Zalaudek et al's study have been compared in Table 2 .

In the present study, two (10\%) lesions had the globular structures over the homogeneous pigmentation, unlike the satellite globules, which are disconnected from the homogeneous part. To our knowledge, this particular pattern has not been demonstrated previously.

It is remarkable that in the present study, four (20\%) lesions showed a parallel ridge pattern, which is the main dermoscopic presentation of early acral melanoma. It

TABLE 2. The frequencies of the dermoscopic findings observed in the present study and Zalaudek et al.'s study [1]

\begin{tabular}{lcccc} 
The studies & The most common colors & Homogenous pattern & Globular pattern & Parallel ridge pattern \\
\hline Elmas et al. $(n=20)$ & Red-black, brown, red & 65 & 55 & 40 \\
Zalaudek et al. $(n=15)$ & Red-black, brown to black & 53.3 & 46.7 & 40
\end{tabular}



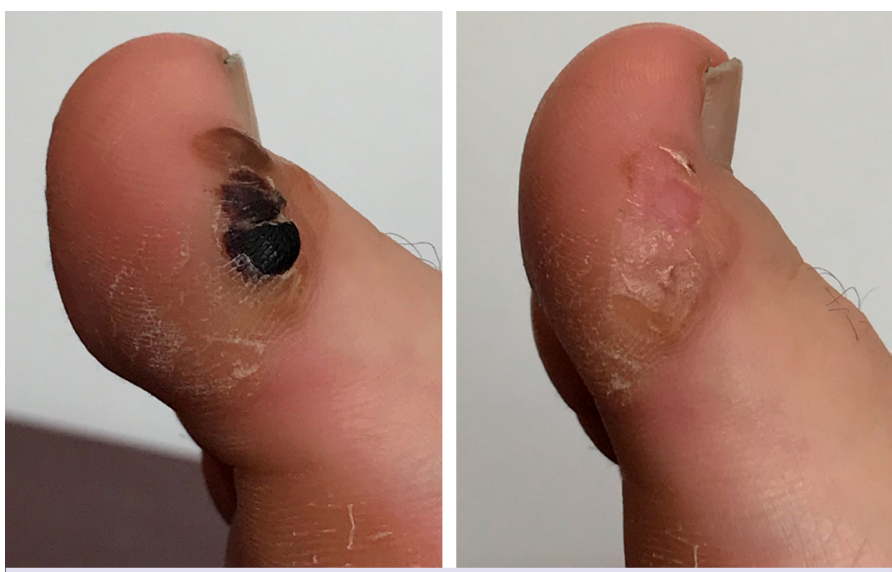

FIGURE 2. A scratch test allows complete removal of pigmentation.

is reported that parallel ridge pattern can also rarely be seen in acral nevi. Zalaudek et al. also demonstrated this pattern in six lesions of SH [1]. They stated that the presence of reddish and grayish coloration can be clues to $\mathrm{SH}$ in such cases. Parallel ridge pattern has also been revealed in two different case reports of $\mathrm{SH}[2,10]$. We hypothesized that parallel ridge pattern observed in $\mathrm{SH}$, may be associated with a particular arrangement of dermal vascular plexus beneath the crista superficialis.

Another common differential diagnosis of $\mathrm{SH}$ is acral nevus. Although acral nevus and $\mathrm{SH}$ may share a similar clinical appearance, it is usually not tricky to separate the two entities dermoscopically as $\mathrm{SH}$ does not exhibit a furrow pattern, which is the main dermoscopic pattern of acral nevi [11]. The presence of the homogenous color, satellite globules and sharp edges are the other useful clues to differentiate $\mathrm{SH}$ from acral nevi.

Zalaudek et al. stated that they confirmed the diagnosis of SH by scratch test, following up and biopsy in four, six, and five patients, respectively [1]. In our study, all the cases underwent to scratch test allowing complete removal of the pigmentation (Fig. 2). We suggest that the scratch test is a useful way to confirm the diagnosis of $\mathrm{SH}$, and the lesions showing dermoscopic features of $\mathrm{SH}$ can easily be confirmed using this practical test to avoid unnecessary invasive procedures.

\section{Conclusions}

To our knowledge, this is the most comprehensive study focusing on the subject addressed in this study. The presence of homogenous red to brown to black pigmentation combined with globular structures may lead to the correct diagnosis. Scratch test may be a minimal invasive diagnostic option to confirm the diagnosis in suspicious cases.

Ethics Committee Approval: This study was approved by the Ahi Evran University, Clinical Research Ethics Committee (Decision date/ number: 13.11.2018/2018-21/177).

Conflict of Interest: No conflict of interest was declared by the authors.

Financial Disclosure: The authors declared that this study has received no financial support.

Authorship Contributions: Concept - OFE; Design - OFE; Supervision - OFE, NA; Fundings - OFE; Materials - OFE; Data collection and/or processing - OFE; Analysis and/or interpretation - NA; Literature review - NA; Writing - OFE; Critical review - NA.

\section{REFERENCES}

1. Zalaudek I, Argenziano G, Soyer HP, Saurat JH, Braun RP. Dermoscopy of subcorneal hematoma. Dermatol Surg 2004;30:1229-32.

2. Uslu U, Heppt F, Erdmann M. Intracorneal Hematoma Showing Clinical and Dermoscopic Features of Acral Lentiginous Melanoma. Case Rep Dermatol Med 2017;2017:3509146. [CrossRef]

3. Yaffee H. Talon noir. Arch Dermatol 1971;104:452. [CrossRef]

4. Ganlupe M. Pinching trauma in black heel. BrJ Dermatol 1967;79:6545. [CrossRef]

5. Juhlin L, Ponten B. Plantar pseudochromidrosis simulating malignant melanoma. Acta Dermatol Venerol 1967;47:255-8.

6. Rufli T. Hyperkeratosis haemorrhagica. Hautarzt 1980;11:606-9.

7. Happler VF. Die Tennisferse (BlackHeel). Z Hautkr 1974;7:285-8.

8. Robertson SJ, Leonard J, Chamberlain AJ. PlayStation purpura. Australas J Dermatol 2010;51:220-2. [CrossRef]

9. Saida T, Oguchi S, Miyazaki A. Dermoscopy: a revolutionary diagnostic approach to the pigmented skin lesions. J Visual Dermatol 2002;1:76-87.

10. Feci L, Fimiani M, Rubegni P. Parallel-ridge pattern on dermatoscopy: observation in a case of purpura traumatica pedis. Dermatol Pract Concept 2015;5:27-9. [CrossRef]

11. Emiroglu N, Cengiz FP, Onsun N. Age and Anatomical Location-Related Dermoscopic Patterns of 210 Acral Melanocytic Nevi in a Turkish Population. J Cutan Med Surg 2017;21:388-94. [CrossRef] 